\title{
Diffraction efficiency of localized holograms in doubly doped $\mathrm{LiNbO}_{3}$ crystals
}

\author{
Christophe Moser, Irena Maravic, Benjamin Schupp, Ali Adibi, and Demetri Psaltis \\ Department of Electrical Engineering, California Institute of Technology, Pasadena, California 91125
}

Received May 10, 2000

\begin{abstract}
The diffraction efficiency of $M$ holograms superimposed in the volume of the recording medium is proportional to $1 / M^{2}$. We present a method, based on nondestructive localized holograms in a doubly doped $\mathrm{LiNbO}_{3}$ crystal, that allows us to also record $M$ holograms in the same volume without an exposure schedule or a diffraction efficiency that has $1 / M$ dependence. We compare experimentally the final diffraction efficiency obtained with the localized and distributed recording methods. (C) 2000 Optical Society of America

OCIS codes: $090.0090,210.0210$.
\end{abstract}

In holographic data storage, pages of information overlap in the volume of the recording medium. As a result of destructive readout of holograms in photorefractive crystals such as $\mathrm{LiNbO}_{3}: \mathrm{Fe}$, holograms are recorded with an exposure schedule to equalize diffraction efficiency, which leads to a final diffraction efficiency that is proportional to $1 / M^{2}$, where $M$ is the number of exposures. We present a technique that does not require an exposure schedule and that can record $M$ holograms with diffraction efficiency following a $1 / M$ dependence. Our technique involves nondestructive readout in doubly doped $\mathrm{LiNbO}_{3}$.

We consider the recording of a hologram by the interference of a reference beam focused by a cylindrical lens and a signal beam, as shown in the inset of Fig. 1. The recording dynamics of such holograms depend strongly on the relative power distribution between the reference and the signal beams. The recording slope $S_{l}$ in photorefractives is given by ${ }^{1}$

$$
S_{l}=\frac{A_{0}}{\tau_{w}} \propto \sqrt{P_{r} P_{s}},
$$

where $A_{0}$ and $\tau_{w}$ are the square root of the saturation diffraction efficiency and the recording time constant, respectively. $P_{r}$ and $P_{s}$ are the power in the reference and the signal beams, respectively. Relation (1) is subject to the constraint that $P_{r}+P_{s}=P_{\text {laser }}$. Maximizing the product in relation (1) subject to this constraint yields $P_{r}=P_{s}$. The square root of saturation diffraction efficiency $A_{0}$ is proportional to the modulation depth $m$ :

$$
A_{0} \propto m=2 \frac{\sqrt{I_{r} I_{s}}}{I_{r}+I_{s}},
$$

where $I_{r}\left(I_{s}\right)$ is the ratio of the power in the reference (signal) and the beam area. This quantity is maximized for equal intensity $I_{r}=I_{s}$, independently of the total power. For equal power in the reference and the signal beams, the recording slope is optimized [relation (1)] but the saturation diffraction efficiency is low because of the resultant low modulation depth [relation (2)]. Conversely, for equal intensities the recording slope is low but the saturation diffraction efficiency is maximized. The difference in dynamics between equal power and equal intensity is due to the large difference between the sizes of the reference and the signal beams.
The following experiment illustrates this dynamic: We use a $90^{\circ}$ cut $\mathrm{LiNbO}_{3}$ crystal doped with 0.01-wt. $\% \mathrm{Fe}_{2} \mathrm{O}_{3}$. The recording beams are generated with a frequency-doubled Nd:YAG laser (Coherent; wavelength, $532 \mathrm{~nm}$ ) that is linearly polarized. A cylindrical lens focuses the reference beam (8-mm diameter, ordinary polarization) in the crystal to a lateral extension of $500 \mu \mathrm{m}\left(1 / e^{2}\right.$ value). The reference beam interferes with a plane-wave signal beam (8-mm diameter, ordinary polarization) as shown in Fig. 1. The total power is distributed into the reference and signal beams by a half-wave plate and a polarizing beam splitter. In the first experiment the reference and the signal beams have equal power. In the second experiment the intensity between the reference and the signal beams is equalized while the total power is kept constant. The result is shown in Fig. 2. As predicted, the curve recorded with equal power has a larger recording slope than the curve recorded with equal intensities and saturates to a much lower diffraction efficiency.

The present authors and others recently demonstrated $^{2}$ the recording of multiple holograms in a doubly doped $\mathrm{LiNbO}_{3}: \mathrm{Fe}, \mathrm{Mn}$ crystal with the

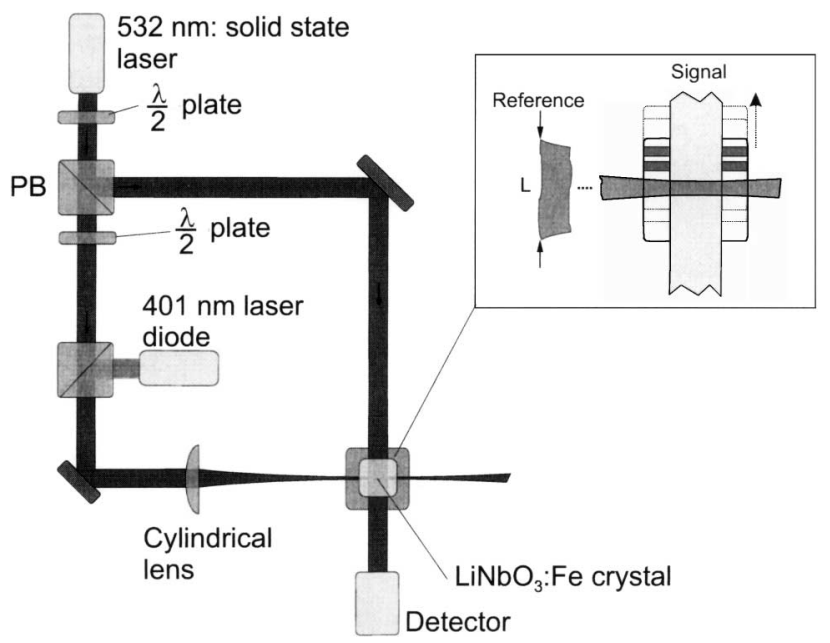

Fig. 1. Recording setup. The half-wave plate positioned before the polarizing beam splitter (PB) distributes the power between the reference and the signal beams. The wave plate is mounted upon a rotation stage for continuous power distribution.

(C) 2000 Optical Society of America 


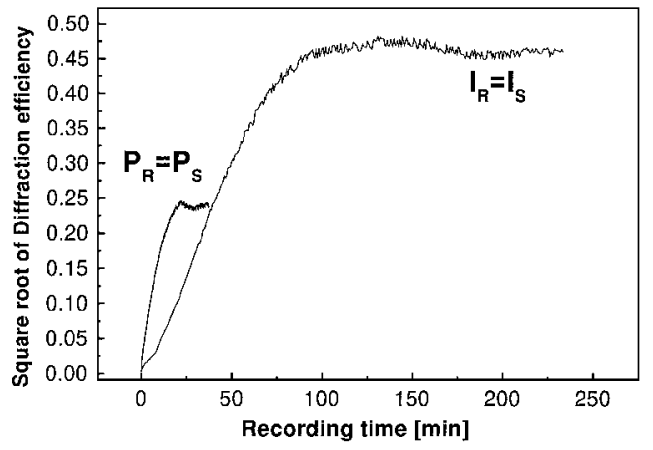

Fig. 2. Recording curves of a localized hologram. The longer curve is recorded with equal intensities in the reference and the signal beams $\left(I_{r}=I_{s}=11.6 \mathrm{~mW} / \mathrm{cm}^{2}\right)$. The other curve is recorded with equal power $\left(P_{r}=P_{s}=\right.$ $3.1 \mathrm{~mW})$. The reference beam is focused to a lateral extension of $500 \mu \mathrm{m}$, and the signal beam is a plane wave of 8-mm diameter.

geometry of Fig. 1. This method works for any gated recorded material. ${ }^{3-7}$ In what follows, we compare the diffraction efficiency of the localized recording method with that of the conventional method of recording superimposed holograms in the same volume.

Let $\alpha$ be the ratio of the power in the reference beam to the total power:

$$
\alpha=P_{r} /\left(P_{r}+P_{s}\right) .
$$

We take the areas of the unfocused reference beam and of the signal beam to be equal. In the following set of equations, the primed variables correspond to the recording with a focused reference beam. The square root of the diffraction efficiency, $\sqrt{\eta^{\prime}}$, of a localized hologram computed as the ratio of the diffracted power to the incident power is given $b^{2}$

$$
\sqrt{\eta^{\prime}}=\sqrt{M} A_{0}^{\prime}\left[1-\exp -\left(t / \tau_{w}{ }^{\prime}\right)\right] .
$$

where $A_{0}^{\prime}$ and $\tau_{w}{ }^{\prime}$ are the saturation diffraction efficiency (measured as the intensity ratio between diffracted and incident beams) and the recording-time constant of the localized recording, respectively. $A_{0}^{\prime}=A_{0}\left(m^{\prime} L^{\prime} / m L\right)$, where $A_{0}$ is the saturation diffraction efficiency for the unfocused reference beam recording (corresponding to $m=1$ ). Equation (4) represents the diffraction efficiency of a single hologram recorded with a focused reference beam that is $M$ times smaller than the original plane-wave reference ( $M=L / L^{\prime}$; Fig. 1 ). $M$ is also equal to the number of localized holograms that can be recorded across a crystal of length $L$. The factor $\sqrt{M}$ that appears in Eq. (4) accounts for the focusing of the reference beam by a factor $M$. Using Eq. (3), and substituting $A_{0}^{\prime}$ and $\tau_{w}{ }^{\prime}$ into Eq. (4), we obtain

$$
\begin{aligned}
\sqrt{\eta^{\prime}}= & 2 A_{0} \frac{\sqrt{\alpha(1-\alpha)}}{1+\alpha(M-1)} \\
& \times\left(1-\exp \left\{-t / \tau_{w}[1+\alpha(M-1)]\right\}\right),
\end{aligned}
$$

where $\tau_{w}$ is the recording-time constant that corresponds to the unfocused reference beam and $\alpha$ takes the value $1 / 2$ for equal power in reference and signal beams. For equal intensities, $\alpha=1 / M+1$.
Substituting $\alpha=1 / M+1$ into Eq. (5), we find that the saturation diffraction efficiency of a single localized hologram recorded with equal intensities is equal to

$$
\eta^{\prime}=A_{0}^{2} / M
$$

where $A_{0}^{2}$ is the saturation diffraction efficiency of a single volume hologram. When $M$ such holograms are recorded side by side in doubly doped $\mathrm{LiNbO}_{3}: \mathrm{Fe}, \mathrm{Mn}$, the efficiency of each hologram is not reduced by the recording of the remaining holograms. This result suggests that, after $M$ strong localized holograms are recorded, the final diffraction efficiency of each localized hologram remains equal to $A_{0}^{2} / M$ [Eq. (6)]. When $M$ volume holograms are superimposed, the final diffraction efficiency is equal to $\eta=(M \# / M)^{2}{ }^{1}$ For recording materials that do not have read-write asymmetry in distributed volume recording [which is the case for $\mathrm{LiNbO}_{3}: \mathrm{Fe}, \mathrm{Mn}$ (Ref. 8)], $M \#=A_{0}$. Therefore the localized recording gains a factor of $M$ in diffraction efficiency compared with distributed recording. The total recording time for the localized recording method can be estimated when it is assumed that each hologram is recorded for two time constants $\tau_{w}{ }^{\prime}$ :

$$
T_{\text {loc total }}{ }^{\prime}=2 M \tau_{w}{ }^{\prime}=M \tau_{w} \simeq M \tau_{e},
$$

where we have assumed that the erasing-time constant $\tau_{e}$ of the distributed volume recording is roughly equal to the writing-time constant $\tau_{w}$. Because the total recording time of the distributed volume method is equal to $\tau_{e} \ln (M)$, the total recording time for the localized holograms is increased by a factor of $\simeq[M / \ln (M)]$.

To verify the $1 / M$ dependence in diffraction efficiency by using localized recording in a persistent material, we experimentally compare the final diffraction efficiency obtained after recording 50 holograms by distributed volume recording and by localized recording in the same doubly doped $\mathrm{LiNbO}_{3}$ crystal. We use a 0.85 -mm-thick crystal doped with 0.075-wt. \% $\mathrm{Fe}_{2} \mathrm{O}_{3}$ and 0.01 wt. $\% \mathrm{MnO}$ in transmission geometry. For localized recording we use a green recording wavelength of $532 \mathrm{~nm}$ with a reference beam focused by a cylindrical lens, yielding a lateral extension of $40 \mu \mathrm{m}\left(1 / e^{2}\right.$ value). The violet sensitizing beam $(401 \mathrm{~nm})$ is copropagating with the green reference beam. The signal has a diameter of $3 \mathrm{~mm}$ at the crystal. Each hologram is recorded up to saturation for a recording time equal to two writing-time constants (green intensity, $100 \mathrm{~mW} / \mathrm{cm}^{2}$; violet intensity, $10 \mathrm{~mW} / \mathrm{cm}^{2}$; extraordinary polarization). The centerto-center spacing between adjacent holograms is $60 \mu \mathrm{m}$. The total spatial multiplexing is done over $3 \mathrm{~mm}$. Figure 3(i) shows the diffraction efficiency of each recorded localized hologram immediately after each recording. An average diffraction efficiency of $2 \times 10^{-2}$ is achieved. Some of the holograms are not recorded well because of instabilities during the long recording time of each hologram (20 min). Figure 3(ii) shows the final diffraction efficiency after the recording of all 50 holograms. It shows that the early holograms have been significantly erased because of recording by the green signal beam and 
(i)

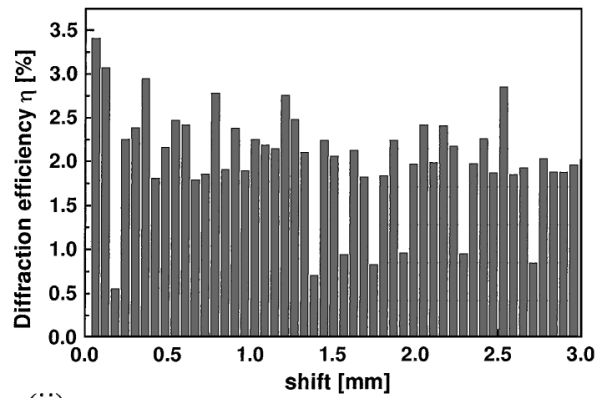

(ii)

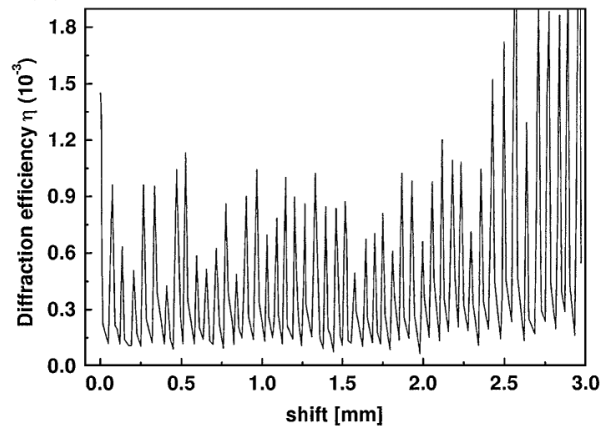

Fig. 3. Diffraction efficiency $\eta$ versus spatial location for (i) $\eta$ of each localized hologram measured immediately after each recording and (ii) $\eta$ measured after recording of 50 holograms.

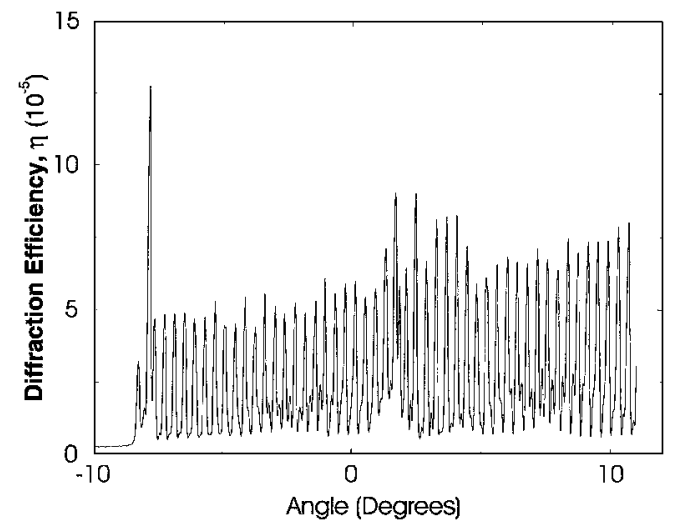

Fig. 4. Diffraction efficiency $\eta$ versus angle for 50 anglemultiplexed holograms.

scattering from the violet sensitizing laser. The average diffraction efficiency is approximately $10^{-3}$.

The distributed volume recording experiment is performed with the same crystal. ${ }^{8}$ Figure 4 shows the diffraction efficiency for 50 angle-multiplexed holograms and ordinary polarization. The measured diffraction efficiency is $5 \times 10^{-5}$ for ordinary polarization; extrapolating from that experiment, we expect a diffraction efficiency for extraordinary polarization of 10 times higher $\left(5 \times 10^{-4}\right)$ because of the higher value of the electro-optic coefficient for extraordinary polarization. The diffraction efficiency obtained with the localized method is twice that obtained with the distributed recording method; theory predicts that it should be a factor of 50 greater.

We can explain this discrepancy as being due to three factors. The first factor is the finite erasing-time constant of the grating stored in the manganese traps for the green signal beam [for Eq. (6) an infinite erasing time constant is assumed]. Because of the long recording time of each localized hologram, the total exposure becomes large enough to erase significant numbers of early holograms. The first recorded hologram is erased only by the signal beam for the remainder of the $M 1$ recording. Experimentally, the ratio of the erasing-time constant to the writing-time constant is 140 at $532 \mathrm{~nm}$. We expect the diffraction efficiency of the first hologram to decrease by a factor of $\exp \left[2\left(M 2 \tau_{u} / \tau_{e}\right)\right]=4.17$ from the saturation diffraction efficiency obtained after initial green erasure. The second factor is the erasure caused by the sensitizing beam on adjacent holograms as a result of scattering from the crystal surface and fanning caused by the long recording time of each localized hologram. This factor varies with the center-to-center spacing of localized holograms. In this experiment we measured a decrease in the diffraction efficiency of adjacent holograms of approximately a factor of 2.5 when the centerto-center spacing was 1.5 times the $1 / e^{2}$ spot size. The third factor is the fact that the localized holograms are not adjacent (20- $\mu \mathrm{m}$ gap between two consecutive localized holograms). This gap causes a loss of a factor of 2.2 in diffraction efficiency. Overall, the saturation diffraction efficiency of each localized hologram is decreased by a factor of 22.9 , which agrees well with values obtained experimentally. All three factors can be reduced or completely eliminated if the properties of the recording material can be improved to reduce erasure at the recording wavelength (this is true for red wavelengths, but recording is slow in the red) and if coated and well-polished crystals are used.

We have shown experimentally that localized hologram recording can yield a larger final diffraction efficiency than distributed volume recording in nonvolatile material (e.g., doubly doped $\mathrm{LiNbO}_{3}$ ). Theoretically the final diffraction efficiency with localized recording is proportional to $1 / M$ instead of to $1 / M^{2}$.

This research was supported by the U.S. Air Force/Rome Laboratory and a Jet Propulsion Laboratory research Hybrid Technology MultiThreaded Architecture project. C. Moser's e-mail address is moser@sunoptics.caltech.edu.

\section{References}

1. F. H. Mok, G. Burr, and D. Psaltis, Opt. Lett. 21, 896 (1996).

2. C. Moser, B. Schupp, and D. Psaltis, Opt. Lett. 25, 162 (2000).

3. H. Guenther, G. Wittmann, R. M. Macfarlane, and R. R. Neurgaonkar, Opt. Lett. 22, 1305 (1997).

4. L. Hesselink, S. Orlov, A. Liu, A. Akella, D. Lande, and R. R. Neurgaonkar, Science 282, 1089 (1998).

5. D. A. Parthenopoulos and P. M. Rentzepis, Science 245, 843 (1989).

6. M. M. Wang, S. C. Esener, F. B. McCormick, I. Okgr, A. S. Dvornikov, and P. M. Rentzepis, Opt. Lett. 22, 558 (1997).

7. K. Buse, A. Adibi, and D. Psaltis, Nature 393, 665 (1998).

8. A. Adibi, K. Buse, and D. Psaltis, Opt. Lett. 24, 652 (1999). 